\title{
Brain Neuroscience Cognitive Effects for Efficient Neurobusiness
}

\author{
Rimmy Chuchra \\ Vertex Infosoft Solutions Private Limited \\ Industrial Area, Mohali, Punjab, India
}

\author{
R. K. Seth \\ Department of Physics \\ DAV University, Jalandhar, Punjab, India
}

\begin{abstract}
Recently, neuroscientists are considering neurobusiness as a mind of the market. A literature survey has been conducted that involves the nature of neurons and communication among themselves. A model based upon cognitive effects of brain for efficient neurobusiness is discussed with the motive to think in a way to help for improving human understanding along with the enhancement of brain intelligence for producing efficient and effective results. The concept of neuroscience, management and engineering based upon neurobusiness may significantly improve the growth of work integration in different domains. The main focus of neurobusiness is to improve the branding, and to develop the individual or unique level of thinking leading to innovative practices in the modern marketing trends. In addition, this concept also provides the best option to choose any newly launched market product through neuromarketing. Consequently, it helps to make customers' search easy for the selection of brand of their choice during shopping.
\end{abstract}

\section{Keywords}

Neurobusiness, Neuromarketing, Brain Model, Digital Marketing, Neurons, Neuron Structure and Learning Platform.

\section{INTRODUCTION}

The word "Brain" may be considered as "Brain Research through Advancing Innovative Neurotech". It (Brain) is a type of neural network physical system that has main function to process data and converts into sensible information. As many neuroscientists studied in their literature, that brain is mainly divided into two main sections; viz. right brain section and left brain section. Each section has its own individual functions. As per the literature, the previous research analysis shows that the working of both males and females is little bit different; even their brain structure is same. In addition to finding of scientists in their latest research in evolutionary theory, a wide range of neurobusiness [1] is expanding in several different disciplines. Correspondingly, they also suggest that there is a wide scope of neuroeconomics, neuromarketing and neuroenterpreurnship in future [1]. The main significance to study the concept of brain in detail is to understand its basic functions, cognitive abilities as well as to observe the level of intelligence in individual personalities $[2$, $3]$. As per the data collected by the neuro researchers in neuro market survey, several techniques are available for analyzing the brain imaging data which helps us to understand the deep structure of neural representation of thoughts $[4,5]$. This concept also facilitates us to change the way of thinking and provide a new innovative platform of learning that further helps to enhance or maximize the business market in terms of neurobusiness.
The aim of this proposed model is to think in a variety of ways for the better understanding of market products in the field of neuromarketing that significantly achieves maximum accuracy in the field of neuromarketing [3]. This new designed concept gives more facilities and much more efficient results to ordinary users (who are customers) than the existing technologies such as digital marketing. In addition, the major benefit to utilize the new concept of neurobusiness is to improve and/or increase the trend of "Mind Marketing" (MM) and also to work on new integrated policy scheme of marketing (IPSM) in which professionals from different fields have equally contributed according to their field of specialization. For example; medical professionals give different theories of brain and neural structure, professionals from management field proposes several methods and ideas how to launch effective marketing policies and the professionals from several engineering disciplines proposes new techniques such as brain imaging techniques for deep understanding of brain functions [4]. With performing such type of collaborative tasks, the market value and demand of product of any organization may be enhanced by following or accepting these latest trends of neurobusiness. This model would extends its help to ordinary people for the selection of any newly launched market products based upon maximum rating and recommended comments during neuromarketing following the new techniques in neurobusiness.

\section{RESEARCH DESIGN}

The research design is based upon neuroscience (specifically cognitive neuroscience) and neurobusiness for the efficient outcome of customers' choice and other business decisions. The highly rated and/or recommended products in the market always remains the choice of the customers. Implementation of neurobusiness provides the entrepreneurs maximum gain while keeping the optimum monetary inputs in the business. This model has taken into consideration the actions and decisions of the customers and entrepreneurs that depends upon logics developed by brain. The communication among neurons is a deciding factor in terms of efficiency of any individual or otherwise. The synergetic interaction between neuroscience and neurobusiness by applying brain model (depends upon activity of one's neurons) is represented in Figure 1.

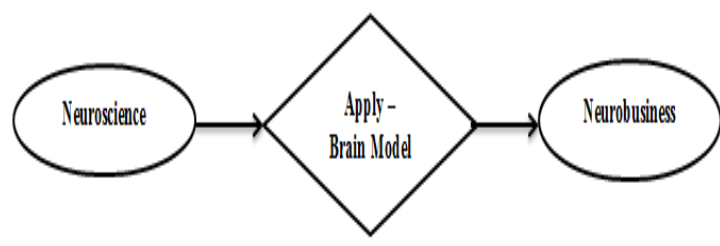

Figure.2.1: Interaction between Neuroscience and Neurobusiness. 
The neuroscience which is directly related to cognitive science makes the business more profitable by utilizing the above demonstrated research design. Therefore both (neuroscience and neurobusiness) direct linkage is more preferable \& most demanding. In addition, neuroscientists may also provide better understanding of cognitive behavior of any individuals who study the structure of neurons and their functions in terms of the active/ passive communication among the neurons.

\section{COGNITIVE MODEL FOR EFFICIENT NEUROBUSINESS}

The roadmap of the model developed for neuromarketing/ neurobusiness is shown in Figure 2.1. Comprising about 86 billion neurons, the brain has three major types of neurons: sensory neurons, motor neurons and interneurons with different functions, to communicate effectively with the other body parts. The configuration of neurons makes the communication among neurons possible by transmission/ reception of signals forms a networking system for various operations/ outcomes.

Neuromarketing has a direct relation with the neuroscience using technologies measuring brain activities (imaging and Scanning etc.) that tells the emotional reaction of human mind to nature of products and other marketing elements such as packaging and advertisements etc.

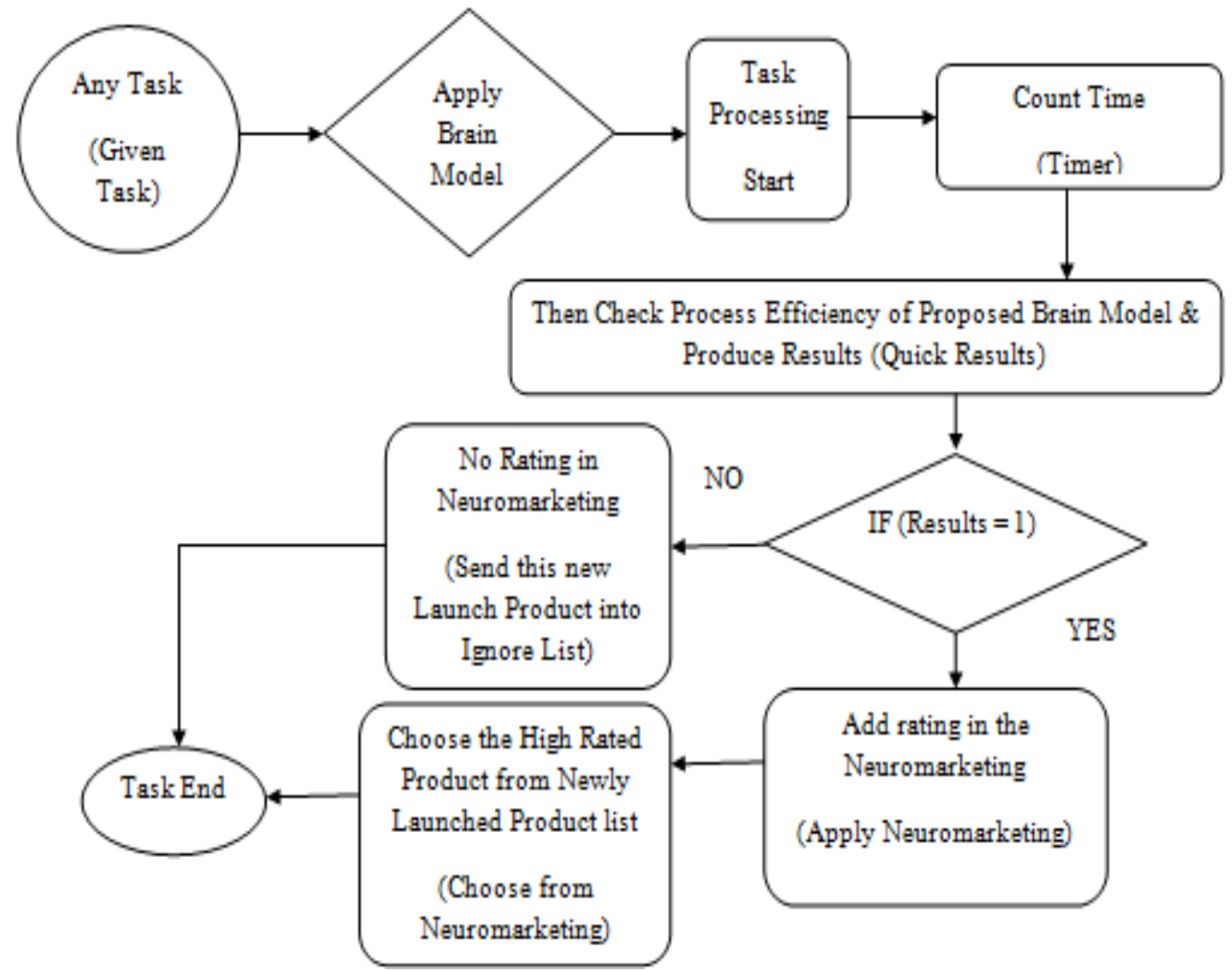

Figure.3.1: A Roadmap for Neuromarketing.

The various steps are described as follows:

Step-1) Input any Given Task.

Step-2) Apply Newly Proposed Brain Model.

Step-3) Start Given Task Processing.

Step-4) Count Time through Timer.

Step-5) Check Efficiency of Proposed Brain Model.

Step-6) IF Results are produced in minimal time then Add Rating in the Neuromarketing.

Step-7) after that, Choose High Rated Newly Launched Product through Neuromarketing.

Step-8) Otherwise put or send into Ignore list.

Step-9) End.

Neurobusiness that encompass neuromarketing has the strength of improving the outcomes in customers and entrepreneurs by evaluating (based upon neuroscience cognitive effects) the insights of the marketing trends.

The nature is reasonable enough by providing different brain models (depending upon nature of neurons). The brain model works depending upon the nature and action of neurons based upon customer(s) and /or entrepreneur(s) running the business. The process of selling/ buying entirely depends upon the cognitive approach based upon the strategies of neurobusiness. The application of brain model gives different results for different individuals. In the representation shown in Figure 3.1, an example of rating of recommendation of product(s) has been taken for taking decision about the product. The rating(s) and recommendation(s) of any product depends upon neuromarketing which further depends upon the nature and quality of product, and upon other strategies of business such as packaging and advertisements etc.

The fast changing business and marketing trends are entirely based upon the new emerging technologies that have impact upon consumer(s) and business personnel due to upcoming new products in the market. Subsequently, neurobusiness/ 
neuromarketing is being employed for making emotional impact of any product upon the customer(s) to enhance the business.

\section{CONCLUSION}

In this article, an effort has been made to develop an understanding of a model neurobusiness on the basis of literature survey of neuroscience and neuromarketing. The diagrammatical representation shows the role, importance or functions with logic for effective neurobusiness by applying brain model (based upon neuroscience of the brain). The benefit of such type of proposed model is to improve the level of thinking that provides a platform to help producing more effective results quickly taking into consideration the human brain intelligence (HBI).The model facilitates to choose a best market product from the newly launched products utilizing the concept of neuromarketing. The authors are of the opinion that the neurobusisness using the concept of neuromarketing would be the Next Generation Business (NGB).

\section{REFERENCES}

[1] Gad Sad and Angela A. Stanton, 2008. Evolutionary Neurobusiness, Aston Center for Human Resources work and Organizational Psychology Research Group.

[2] Ghadiri.A and Habremacher.A, 2012. Neuroscience for Business - A journey through the brain for Business Leaders, Neuroleadership.

[3] Robert Turner and Terry Jones, March 2003. Techniques for Imaging Neuroscience, British Medical Bulletin.

[4] Paul M. Thompson, 2002. Bio-Informatics \& brain imaging: Recent Advances \& Neuroscience Applications, Thesis.

[5] Karim S. Kassam and Amanda R. Marker, Identifying Emotions on the basis of Neural Activation, In Press, PLOS One, Camegle Mellon University. 